\title{
Modelagem numérica da madeira Corymbia citriodora ao ensaio de cisalhamento iosipescu
}

\author{
Luciano Rossi Bilesky ${ }^{1 \star}$, Cláudio De Conti ${ }^{2}$, Priscila Roel de Deus ${ }^{3}$ \\ ${ }^{1}$ Tecnologia em Agroindústria e Tecnologia em Silvicultura, Faculdade de Tecnologia de Capão Bonito, Capão Bonito, SP, \\ Brasil. \\ ${ }^{2}$ Departamento de Engenharia de Energia, UNESP, Rosana, SP, Brasil. \\ ${ }^{3}$ Tecnologia em Silvicultura, Faculdade de Tecnologia de Capão Bonito, Capão Bonito, SP, Brasil.
}

\begin{abstract}
RESUMO Os ensaios normatizados para a determinação das propriedades mecânicas ao cisalhamento da madeira mostram valores subestimados por não apresentarem um estado de cisalhamento puro. O ensaio de cisalhamento Iosipescu foi proposto inicialmente para compósitos artificiais e tem sido adaptado para a utilização em outros materiais. Neste trabalho foi realizada a simulação numérica pelo método de elementos finitos com o uso do software ANSYS para a verificação do comportamento da madeira de Corymbia citriodora ao cisalhamento no plano de simetria LR. O ensaio apresentou bom comportamento mostrando que os campos de tensão na região de colagem do extensômetro foram homogêneos, assim como os campos de deslocamento, podendo então ser aplicado à referida madeira.
\end{abstract}

Palavras-chave: mef (método de elementos finitos), tensões, shear block test.

\section{Numerical modeling wood Corymbia citriodora to shear iosipescu test}

\begin{abstract}
The standardized tests for the determination of the mechanical properties of wood shear have values that are underestimated because they do not present a pure shear state. The Iosipescu shear test was initially proposed for artificial composites and has been adapted in others materials. In this work, a numerical test was performed using the finite element method using the ANSYS software for the control of Corymbia citriodora wood at planar LR symmetry shear. The test together showed that the tensile fields in the extensor extension region were homogeneous, as were the displacement fields, and could be applied to the wood.
\end{abstract}

Keywords: FEM, stress, shear block test.

\section{Introdução}

A madeira por ser um material biológico, renovável e permitir a sua produção e emprego de forma sustentável, tem despertado o interesse de técnicos e engenheiros para a sua aplicação nos mais variados setores, em especial no setor da construção civil, tanto como material estrutural, quanto de acabamento.
Devido a sua heterogeneidade, suas propriedades e comportamentos mecânicos apresentam grande variabilidade devido à complexidade do material.

O desempenho mecânico está entre as propriedades mais relevantes da madeira devido a sua importância na aplicação como material estrutural. Dentre estas propriedades, o cisalhamento está entre os mais visados para a otimização e aplicação da madeira em estruturas. 
Diversos documentos normativos como o EN 408 (2002), ASTM D143-94 (1994) e NBR 7190 (1997) estabelecem as prescrições para a condução do ensaio de resistência característica ao cisalhamento. Todos estes ensaios baseiamse no "shear block test" como padrão, modificando apenas alguns parâmetros como o carregamento e as dimensões do corpo de prova.

Apesar de normalizados, estes ensaios apresentam valores subestimados (Garrido, 2004) por apresentar sobreposição de tensões na região cisalhante do corpo de prova "shear block test”. Bilesky (2017) demonstrou através de simulação numérica pelo método dos elementos finitos que estes valores podem assumir uma intensidade muito inferior à do cisalhamento puro ocorrido por tensões de cisalhamento homogêneas.

No intuito de propiciar um estado de cisalhamento puro, outros ensaios têm sido estudados, entre eles o ensaio de tração off-axis, o ensaio de arcan e o ensaio iosipescu. O ensaio iosipescu é normalizado pela ASTM D5379-93, inicialmente proposto para compósitos artificiais ortotrópicos e que vem sido adaptado para o uso em madeiras (XAVIER et al., 2003), apresentando bons resultados para a determinação das propriedades mecânicas ao cisalhamento.

O presente estudo tem como objetivo a verificação do comportamento da madeira de Corymbia citriodora ao cisalhamento através da simulação numérica pelo método dos elementos finitos ao ensaio iosipescu, com o estudo da homogeneidade dos campos de tensão e deslocamentos na área cisalhante no plano de ortotropia $L R$.

\section{Material e Métodos}

O ensaio iosipescu consiste em um corpo de prova com uma geometria prismática com um entalhe em " $V$ " em $45^{\circ}$ de ângulo no seu centro, como pode ser observado na Figura 1.
Para a solicitação da força no corpo de prova é utilizado o sistema de amarras "Wyoming" modificado, acoplada numa máquina de ensaios universal, presente na Figura 2.

No centro do corpo de prova a medida das deformações é extraída a partir de extensômetros podendo então ser calculada a tensão de cisalhamento $\sigma_{6}$ pela expressão 1 :

$$
\sigma_{6}=\frac{P}{A}
$$

Em que $P$ representa a força total aplicada pela célula de carga e $A$, a área da seção transversal reduzida do corpo de prova iosipescu.

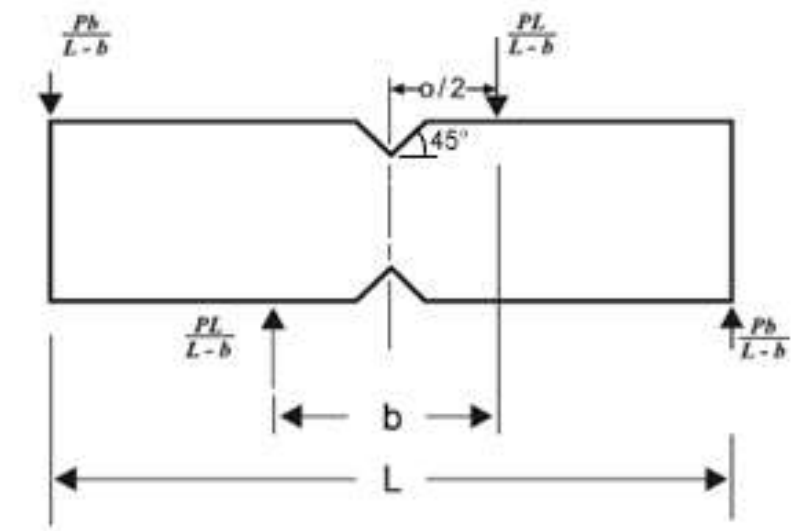

Diagrams de corpo bive

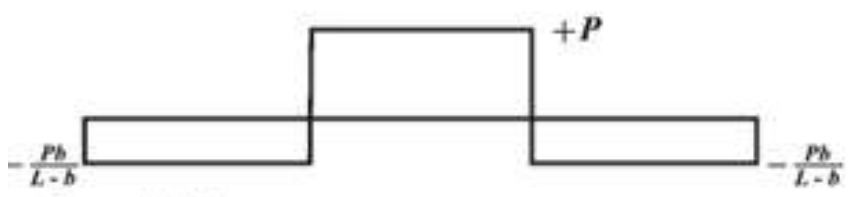

Digrama de ciuatharieng

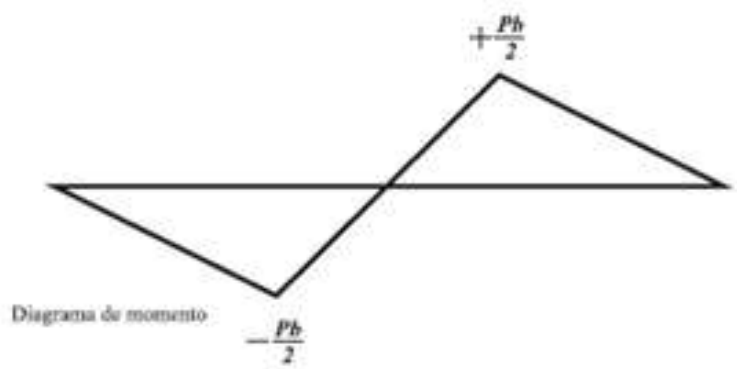

Figura 1 - Diagrama do corpo livre, com o diagrama de cisalhamento e do momento fletor (GONÇALVES et al., 2009).

Figure 1 - Scheme of the free body, with the shear diagram and the bending moment (GONÇALVES et al., 2009). 
Para a simulação numérica do ensaio iosipescu pelo método dos elementos finitos foi utilizado o software comercial ANSYS $9^{\circ}$.

As dimensões do corpo de prova para a construção do modelo numérico estão expressas na Figura 3, sendo as mesmas propostas pela ASTM D5379-93.
As constantes de engenharia módulo de elasticidade $(E)$, coeficiente de Poisson $(v)$ e módulo de elasticidade na seção transversal do plano $L R(G)$, nas direções longitudinal $(L)$, radial $(R)$ e tangencial $(T)$ da madeira de Corymbia citriodora utilizadas para a construção do modelo numérico são as fornecidas por Ballarin e Nogueira (2003).

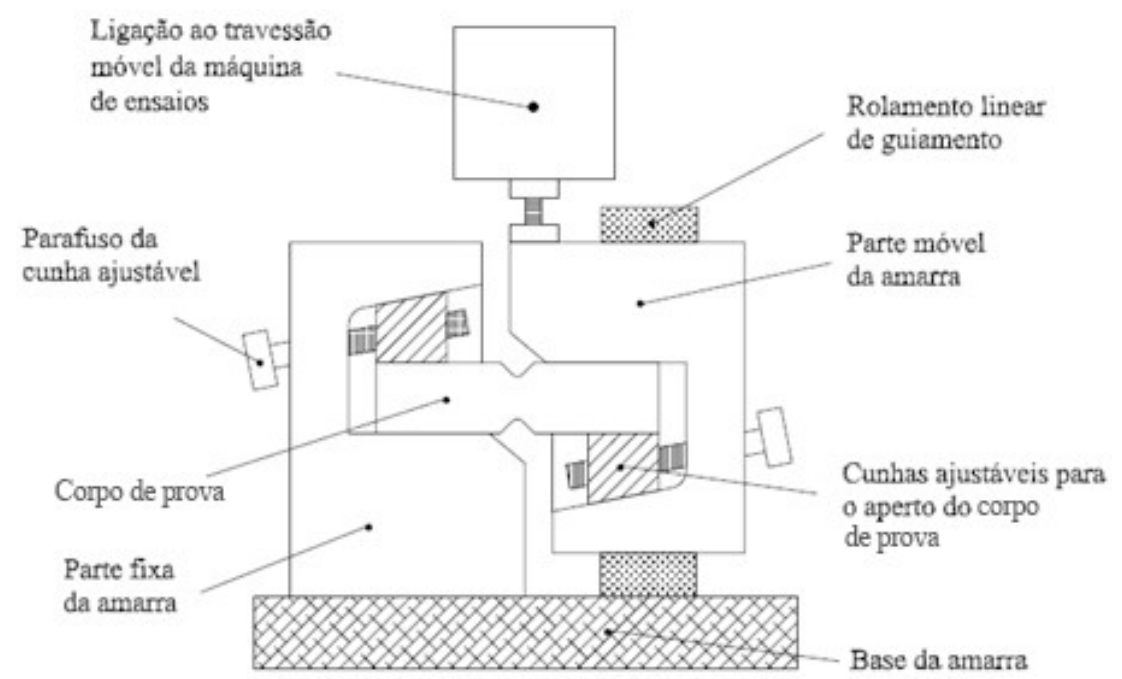

Figura 2 - Esquema da amarra associada ao ensaio iosipescu (ASTM D5379-93).

Figure 2 - Mooring scheme associated with the iosipescu test (ASTM D5379-93).

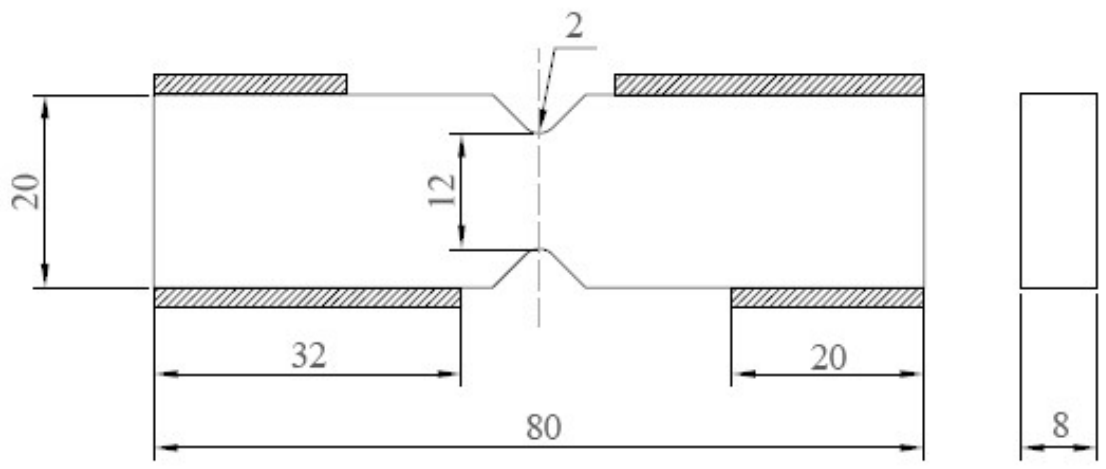

Figura 3 - Dimensões do corpo de prova iosipescu em mm segundo a ASTM D5379-93.

Figure 3 - Dimensions of the specimen iosipescu in mm proposed by ASTM D5379-93.

Tabela 1 - Constantes de engenharia de Corymbia citriodora.

Table 1 - Engineering constants of Corymbia citriodora.

\begin{tabular}{llll}
\hline Corymbia citrodora & & \\
\hline $\mathrm{E}_{\mathrm{L}}$ & $16981 \mathrm{MPa}$ & $\mathrm{G}_{\mathrm{LR}}$ & $861 \mathrm{MPa}$ \\
$\mathrm{E}_{\mathrm{R}}$ & $1825 \mathrm{MPa}$ & $\mathrm{S}_{11}$ & $0,058.10^{-12}$ \\
$v_{\mathrm{LR}}$ & 0,23 & $\mathrm{~S}_{12}$ & $0,014.10^{-12}$ \\
$v_{\mathrm{LT}}$ & 0,48 & $\mathrm{~S}_{22}$ & $0,548.10^{-12}$ \\
$v_{\mathrm{RT}}$ & 0,70 & $\mathrm{~S}_{66}$ & $1,161.10^{-12}$ \\
\hline
\end{tabular}

E - módulo de elasticidade; $v$ - coeficiente de Poisson; G - módulo de cisalhamento; S - coeficientes da matriz de elasticidade. Coeficiente: $\mathrm{L}$ - longitudinal; $\mathrm{R}$ - radial e $\mathrm{T}$ - transversal.

(Ballarin e Nogueira, 2003) 
Para a simulação numérica foi selecionado o elemento PLANE 182 da biblioteca de elementos ANSYS $9^{\circ}$, por ser um elemento que possui os graus de liberdade necessários para a presente simulação.

As condições de contorno aplicadas ao modelo numérico estão expressas na Figura 4, onde $u_{i}$ representa os deslocamentos na direção $i$ e $\delta$ correspondente a um deslocamento de $0,5 \mathrm{~mm}$.

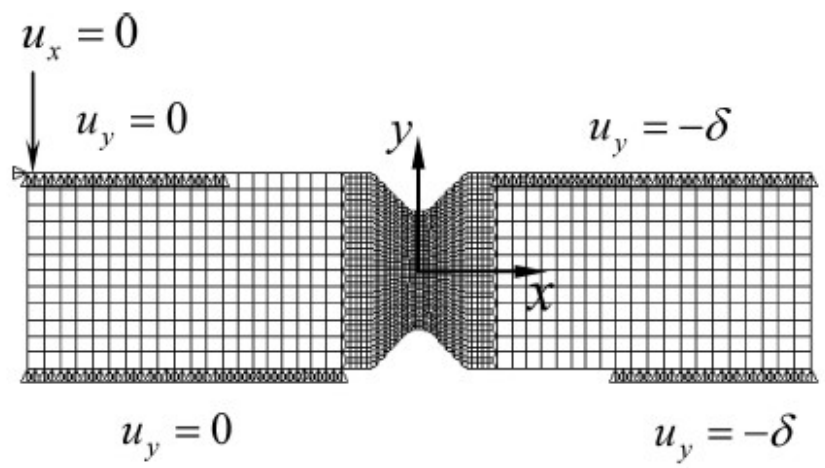

Figura 4 - Condições de contorno aplicadas ao modelo numérico adaptado de Xavier et al. (2003).

Figure 4 - Contour conditions applied to the numerical model adapted from Xavier et al. (2003).

\section{Resultados e Discussão}

A malha do modelo numérico selecionada é construída de 10.600 elementos e 32.275 nós, a partir de análise de convergência com o critério da estabilização dos deslocamentos com o aumento da densidade da malha, sendo selecionada aquela com menor densidade quando estabilizados os resultados (Figura 5).

A Figura 6 apresenta o campo de deslocamentos $\varepsilon_{x}$ obtidos através da simulação numérica, no qual pode-se observar um paralelismo nas nuances das cores que representam os deslocamentos, evidenciando um deslocamento homogêneo e proporcional nas regiões de máximo deslocamento (MX) e região de mínimo deslocamento $(\mathrm{MN})$.
No centro do corpo de prova, onde denominamos de região cisalhante, encontram-se os menores valores de deslocamento dos campos $\varepsilon_{x}$ com ordem de grandeza E- $07 \mathrm{~m}$ e gradualmente e proporcionalmente tanto para cima, quanto para baixo os valores dos deslocamentos possuem acréscimos graduais.

Na Figura 7 estão expressos os campos de deslocamentos $\varepsilon_{y}$ do modelo do corpo de prova obtido através da simulação numérica. $\mathrm{O}$ centro do corpo de prova apresenta os menores valores de deslocamentos e na direção horizontal que demonstra ocorrer o acréscimo gradual e proporcional dos campos de deslocamento $\varepsilon_{y}$ tanto no sentido da direita quanto no sentido da esquerda.

Pode-se observar que os campos de deslocamentos $\varepsilon_{x}$ na região de ruptura do corpo de prova são uniformes assim como os campos de deslocamento $\varepsilon_{y}$, produzindo uma deformação homogênea na região cisalhante, propiciando o princípio de Saint-venant.

O comportamento de homogeneidade nos campos de deslocamento $\varepsilon_{x}$ e $\varepsilon_{y}$ pode ser observado em Xavier et al. (2003) para madeira de Pinus pinaster Ait. no plano de $L R$ e também em material anisotrópico simulado em Fakoor e Khansari (2018), o qual demonstra a mesma tendência de deslocamentos graduais, efeito este desejável para a obtenção de estado de cisalhamento puro na região cisalhante.

A Figura 8 apresenta o campo de tensão $\sigma_{x}$, obtido através do modelo numérico em que a mesma ordem de grandeza é observa em todo o corpo de prova, excetuando-se os pontos onde as condições de contorno mostradas na Figura 4 são aplicadas, tanto na fixação, quanto no deslocamento. Nessas regiões são encontradas as maiores tensões, mas este fenômeno é esperado, pois as condições de contorno representadas na análise são bastante constritivas. 


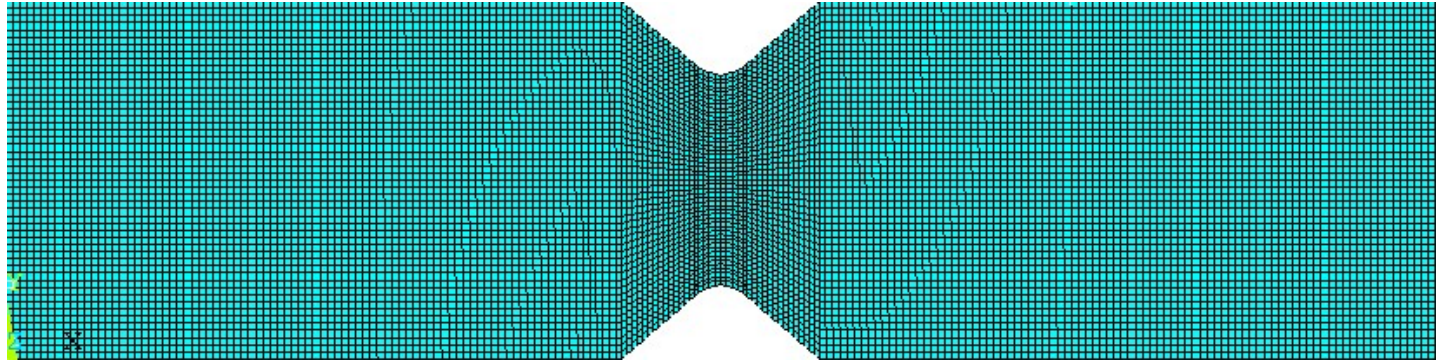

Figura 5 - Malha do modelo numérico do corpo de prova iosipescu.

Figure 5 - Mesh of the numerical model of the iosipescu specimen.

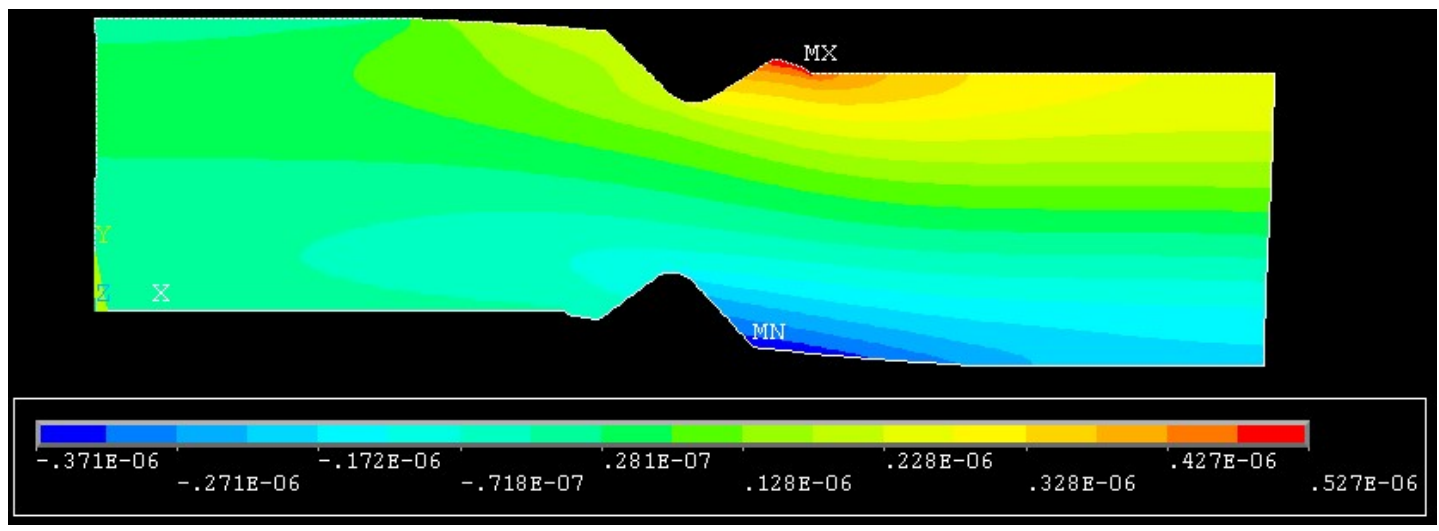

Figura 6 - Campos de deslocamentos $\varepsilon_{x}$ do modelo do corpo de prova iosipescu em $\mathrm{m}$.

Figure 6 - Fields of displacement $\varepsilon_{x}$ of the test specimen model iosipescu in m.

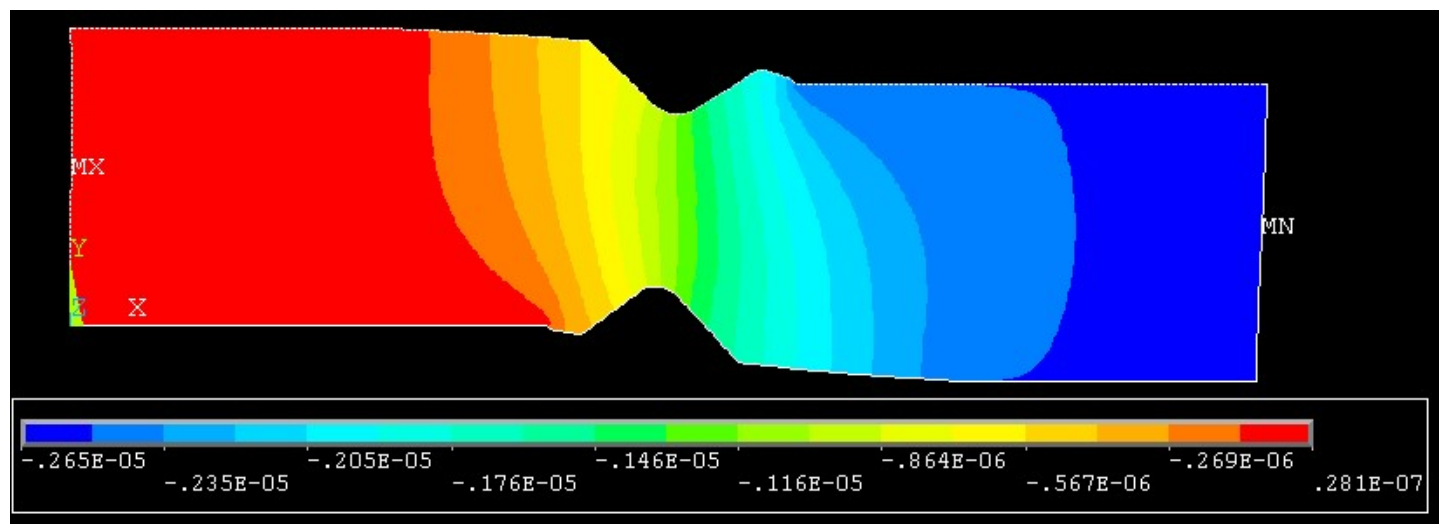

Figura 7 - Campos de deslocamentos $\varepsilon_{y}$ do modelo do corpo de prova iosipescu em $\mathrm{m}$.

Figure 7 - Fields of displacement $\varepsilon_{y}$ of the model of the specimen iosipescu in $\mathrm{m}$.

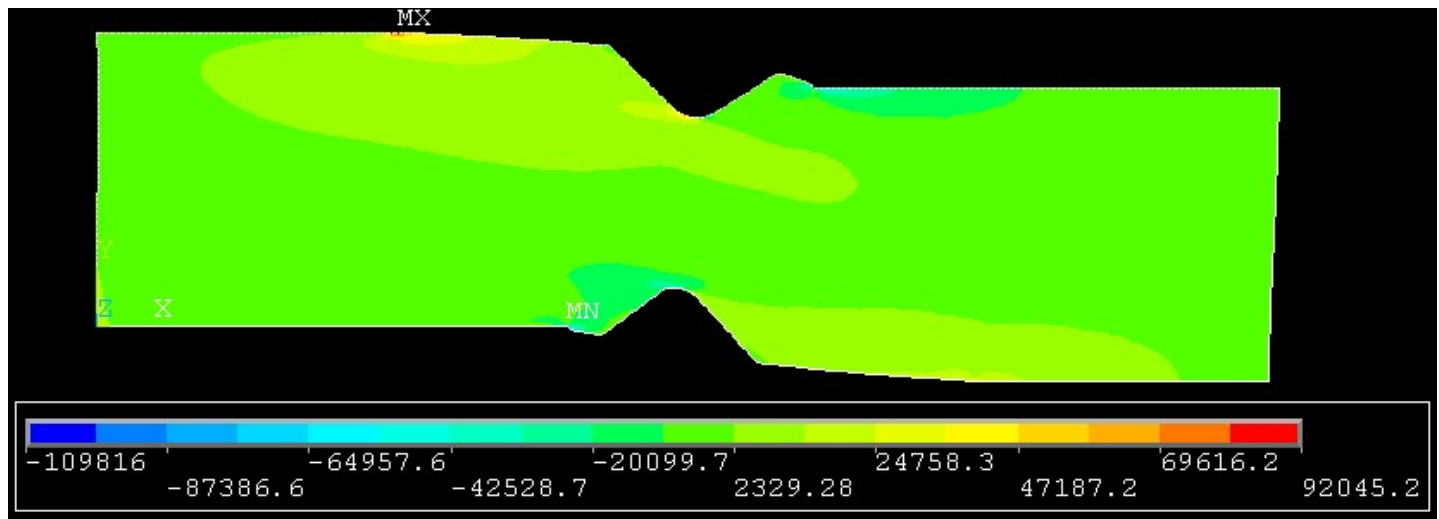

Figura 8 - Campos de tensão $\sigma_{x}$ do modelo do corpo de prova iosipescu em $\mathrm{Pa}$.

Figure 8 - Fields of stress $\sigma_{x}$ of the model of the test specimen iosipescu in $\mathrm{Pa}$. 
Na Figura 9 que representa os campos de tensão $\sigma_{y}$ do modelo numérico, apresenta a mesma homogeneidade das tensões encontradas nos campos de tensão $\sigma_{x}$, com a mesma ordem de grandeza em todo o corpo de prova e tensões sobressalentes nas regiões constritivas das condições de contorno.

Os campos de tensão $\sigma_{x y}$ apresentados no modelo numérico na Figura 10 demonstram a homogeneidade das tensões na região do extensômetro do corpo de prova, efeito análogo ao determinado por Xavier et al. (2004).

Pode-se verificar com o auxílio da Figura 11 que os campos de tensão de $\sigma_{x y}$ são uniformes e uniaxiais em praticamente todo o corpo de prova, principalmente na região cisalhante de forma que se propicia um estado de tensão homogêneo tendendo desta forma a ruptura na região com menor área sem a sobreposição de tensões permitindo a medida do verdadeiro módulo de cisalhamento em um estado de tensão cisalhante puro.

A curva representada no gráfico da Figura 11 possui a mesma característica da curva obtida por Fakoor e Khansari (2018) para material anisotrópico simulado, o qual evidenciase uma menor tensão nas extremidades longitudinais do corpo de prova e um acréscimo na região central de colagem do extensômetro.

A diferença de tensão $\sigma_{x y}$ ao longo do eixo $x$ do corpo de prova não ultrapassa $20 \%$ e por acentuar-se no centro proporciona a ruptura em sua região central, onde o extensômetro deve ser colado.

Bilesky et al (2017) realizou a simulação numérica do ensaio de cisalhamento normatizado NBR 7190 (1997) com a madeira de Corymbia citriodora e verificou que os campos te tensão $\sigma_{x y}$ na área cisalhante podem obter uma diferença de

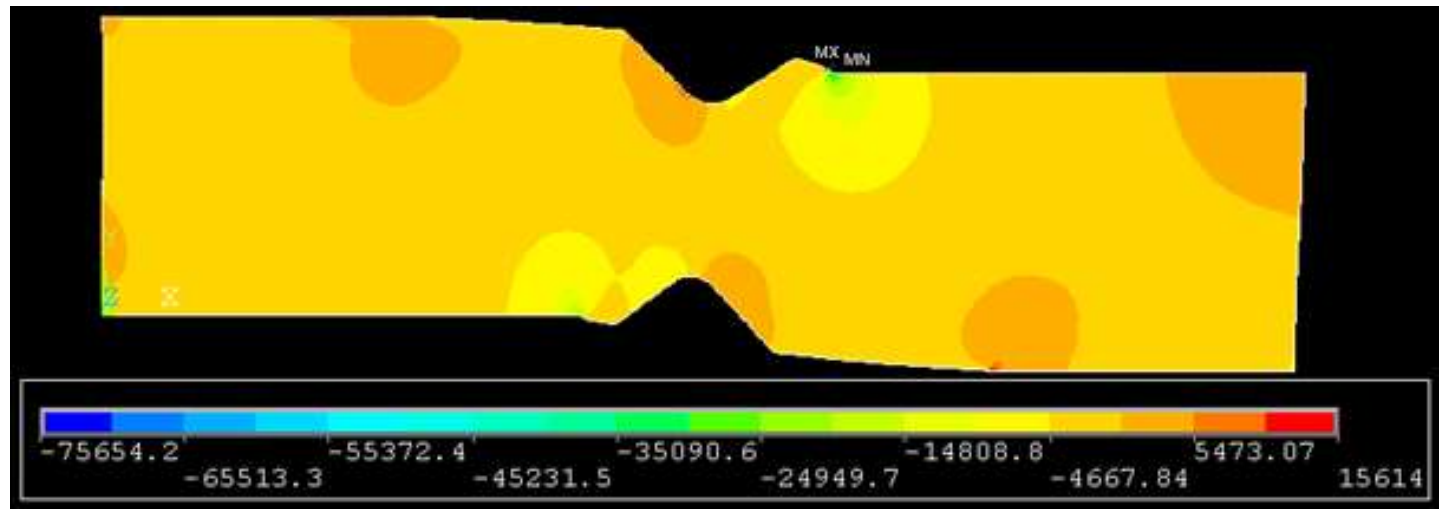

Figura 9 - Campos de tensão $\sigma_{y}$ do modelo do corpo de prova iosipescu em $\mathrm{Pa}$.

Figure 9 - Fields of stress $\sigma_{y}$ of the model of the test specimen iosipescu in $\mathrm{Pa}$.

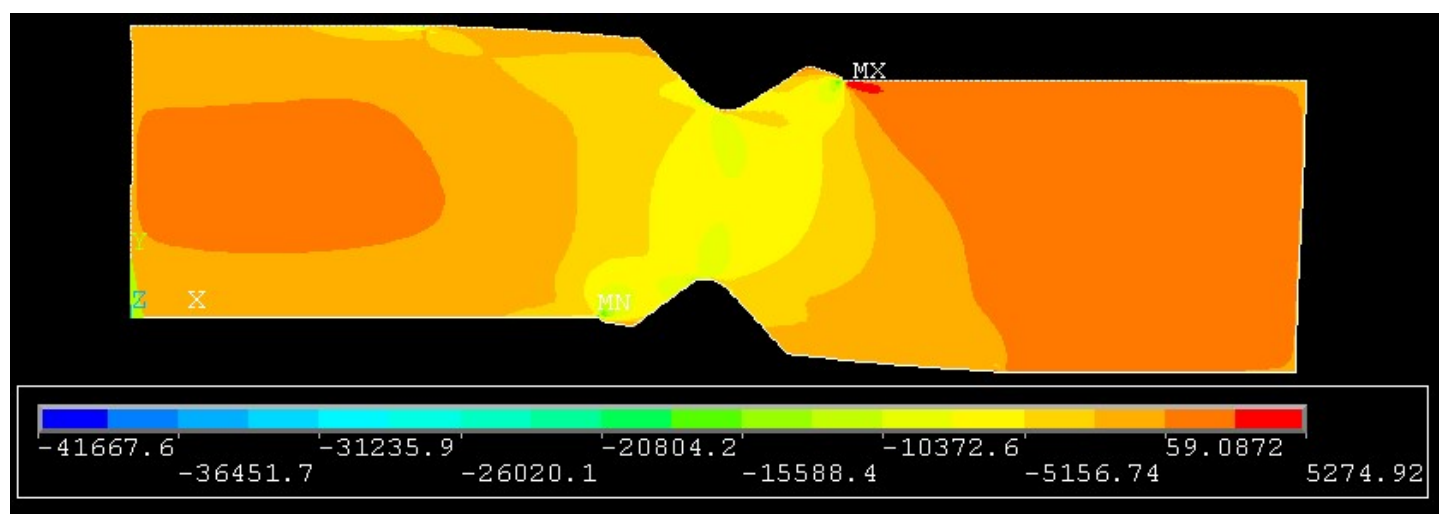

Figura 10 - Campos de tensão $\sigma_{x y}$ do modelo do corpo de prova iosipescu em $\mathrm{Pa}$.

Figure 10 - Fields of stress $\sigma_{x y}$ of the model of the test specimen iosipescu in $\mathrm{Pa}$. 


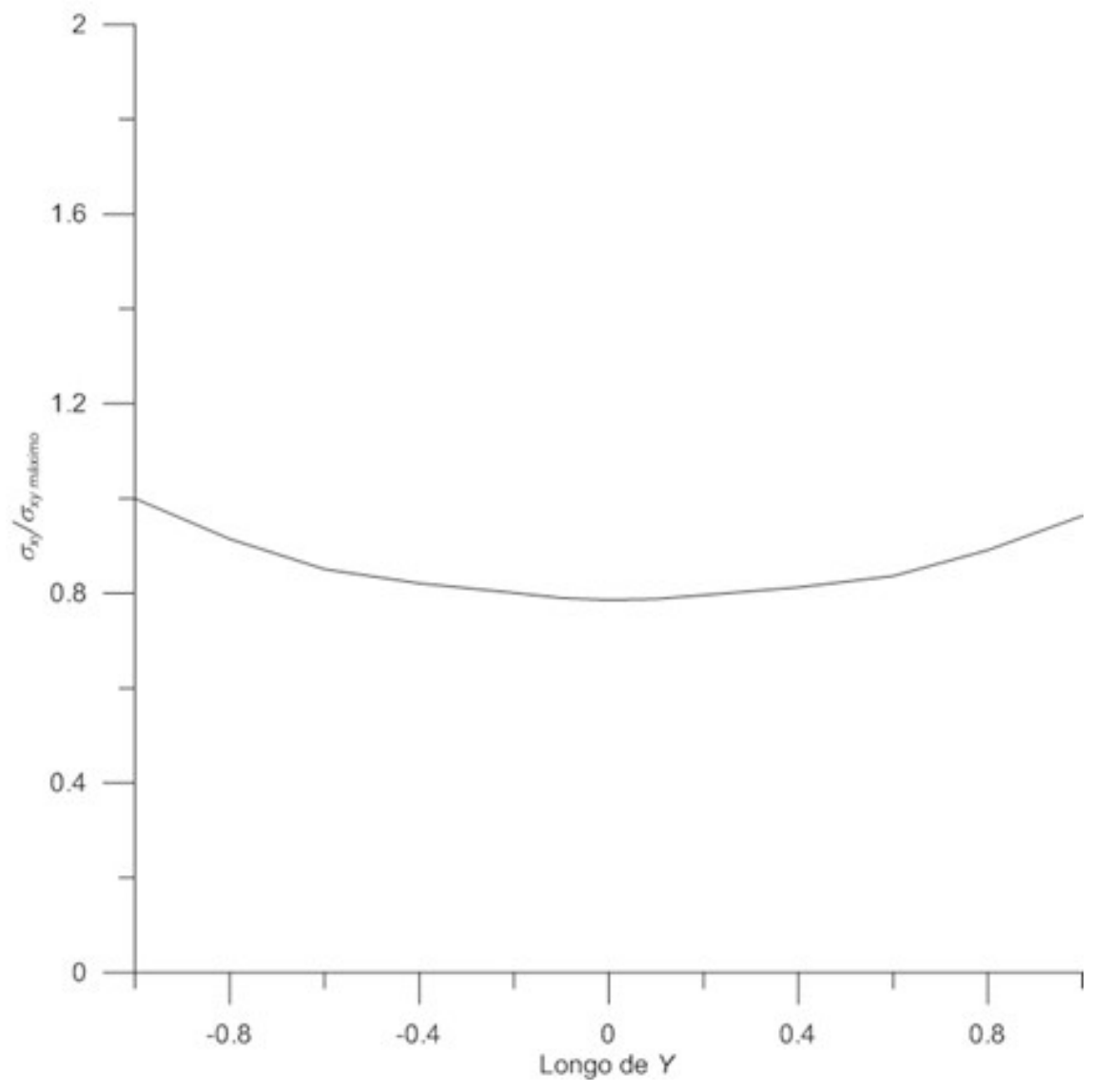

Figura 11 - Gráfico das tensões normalizadas $\sigma_{x y} / \sigma_{x y \text { máximo }}$ no centro do corpo de prova na direção $Y$.

Figure 11 - Graph of the normalized stresses $\sigma_{x y} / \sigma_{x y}$ maximum in the center of the specimen in the $Y$ direction.

até $67 \%$ ao longo da direção $x$ e de até $76 \%$ ao longo da direção

$y$, diferença que supera em até $56 \%$ aos verificados no gráfico

da Figura 11, evidenciando que o ensaio iosipescu pode proporcionar um estado de cisalhamento mais homogêneo quando comparado ao "shear block test".

\section{Conclusões}

A simulação numérica pelo método dos elementos finitos do ensaio iosipescu aplicado à madeira de Corymbia citriodora se apresentou adequada para a determinação das propriedades ao cisalhamento no plano de ortotropia $L R$.

Os campos de deslocamento e os campos de tensão propiciam tensões uniaxiais e homogêneas na região de colagem do extensômetro, desta forma a tensão de cisalhamento nesta região para esta espécie pode ser considerada pura.

\section{Referências}

AMERICAN SOCIETY FOR TESTING AND MATERIALS. ASTM D143-94: Standard methods of testing small clear specimens of timber. Philadelphia, PA, USA, 1994.

ASSOCIAÇÃO BRASILEIRA DE NORMAS TÉCNICAS. NBR 7190: Projeto de estruturas de madeira. Rio de Janeiro, 1997.

BALLARIN, A. W.; NOGUEIRA, M. Caracterização elástica da madeira de Eucalyptus Citriodora. Revista Cerne, 9(1): 66-80, 2003.

BODIG, J.; JAYNE, B.A. Mechanics of wood and wood composites. 2. ed., Florida. Krieger Publishing Company, $1982,712 \mathrm{p}$ 
BILESKY, L. R.; DE CONTI, C, DEUS, P. R. Numerical Simulation of the Shear Resistence Test Proposed by NBR 7190 (1997) for a Wood of Corymbia Citriodora. Mechanics, Materials Science \& Engineering, 11. 2017.

BILESKY, L. R. Simulação numérica do comportamento da madeira de Eucalyptus citriodora ao cisalhamento através do ensaio de tração off-axis. Dissertação (Mestrado em Eng. Mecânica) - Univ. Estadual Paulista - UNESP, Guaratinguetá, 2010.

EN 408. Timber structures - Structural timber and glued laminated timber - Determination of some physical and mechanical properties. European Committee for Standardization, Office for Official Publications of the European Communities, Luxembourg, 2002.

FAKOOR, M.; KHANSARI, N. M. A New Approach for Investigation of Mode II Fracture Toughness in Orthotropic Materials. Latin American Journal of Solids and Structures, 15(3), 2018.

GONÇALVES, V. O.; PARDINI, L. C.; GARCIA, K.; ANCELOTTI JUNIOR, A. C.; BEZERRA, E. M. Resistência ao cisalhamento Iosipescu em compósitos de fibras de carbono e de vidro com resina epóxi. Journal of Aerospace Technology and Management. 1 (1): 49-53, 2009.

XAVIER, J.; OLIVEIRA, J.; MORAIS, J.; CAMANHO, P.P.; PIERRON, F. Measurement of the shear modulus of wood P. pinaster Ait. by the iosipescu test: numerical aspects. Livro de Actas do VII Congresso Nacional de Mecânica Aplicada e Computacional. 1, 2003.

XAVIER, J. C.; GARRIDO, N. M.; OLIVEIRA, M.; MORAIS, J. L.; CAMANHO, P. P.; PIERRON, F. A comparison between the Iosipescu and off-axis shear test methods for the characterization of Pinus Pinaster Ait. Composites: Part A. 35: 827-840, 2004. 